R.T. Schilizzi ${ }^{1}$, B.F. Burke ${ }^{2}$, R.S. Booth ${ }^{3}$, R.A. Preston ${ }^{4}$, P.N. Wilkinson ${ }^{5}$, J.F. Jordan ${ }^{4}$ E. Preuss ${ }^{6}$, D. Roberts 7

1 Netherlands Foundation for Radio Astronomy, Dwingeloo, NL

2 Massachusetts Institute of Technology, Cambridge, Mass. USA

${ }^{3}$ Onsala Space Observatory, Onsala, Sweden

4 Jet Propulsion Laboratory, Pasadena, Calif. USA

5 Nuffield Radio Astronomy Laboratories, Jodrell Bank, UK

6 Max-Planck-Institut für Radioastronomie, Bonn, FRG

7 Brandeis University, Waltham, Mass. USA

\title{
SPACE VLBI AND QUASAT
}

Preston et al (1976) and Burke (1982, these proceedings) have long extolled the virtues of launching a radio telescope into space to increase VLBI baseline lengths and thus angular resolution, and to provide a much enhanced image formation capability. The scientific motivation for this has been covered in a number of memoranda referenced by Burke in these proceedings, and by Anderson et al (1982). Efforts to mobilise western astronomical support for space VLBI met with success in late 1982 at a meeting of US and European radio astronomers in Toulouse, France, at which a decision was taken to propose a joint mission to ESA and NASA. Shortly thereafter, a formal proposal was made to ESA (Anderson et al 1982) for a free flying satellite in an elliptical orbit out to $15000 \mathrm{~km}$ from the Earth, designed to observe in concert with the major ground-based VLBI networks and arrays. The mission, dubbed QUASAT, was received favourably in both ESA and NASA, with the result that formal Assessment Studies are scheduled to begin in both agencies in October 1983.

\section{THE ADVANTAGES OF ORBITING ANTENNAS FOR VLBI}

A space VLBI system (Figure 1), and QUASAT in particular, offers several crucial advantages over ground-based VLBI (see also Morgan et al 1982, Preston et al 1983):

1) angular resolution - Baselines to QUASAT are three times longer than the maximum baselines on Earth, thus providing at least a factor of three increase in angular resolution (or an order of magnitude decrease in the area of the equivalent "seeing disk"). "Super-resolution" should increase the resolution by a further factor (see point $2 b$ below).

$$
407
$$

\footnotetext{
R. Fanti et al. (eds.), VLBI and Compact Radio Sources, 407-414.

(C) 1984 by the IAU.
} 


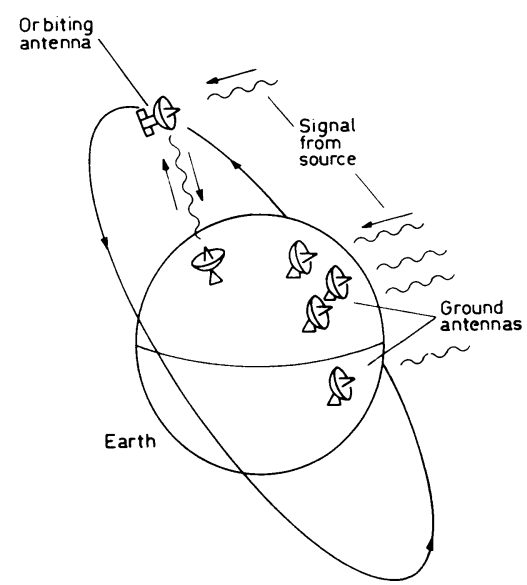

Figure 1 Schematic diagram of QUASAT VLBI system

2) filled $u-v$ plane - The relative motion of QUASAT and the Earth provides for exceptionally dense coverage of the aperture plane of the interferometer system. As long as the QUASAT orbital period is not commensurate with the Earth's rotational period, each pass of the satellite contributes additional spatial frequencies in the $u-v$ plane. This is important in reducing the usual ambiguities and uncertainties in image restoration. Particular points to note in this regard are:

a) The field of view governed by the size of "holes" in the u-v plane is substantially enlarged compared with that of a ground-based array alone. The other factors affecting the field of view are discussed later in this paper.

b) Super-resolution becomes possible. In the standard image restoration algorithms, the final map is smoothed with a gaussian function whose FWHM is of the order of $1 / r_{\max }$ radians where $r_{\max }$ is the maximum baseline length in wavelengths. This is a very caxutious approach, corresponding as it does to weighting down the contribution of the data from the longest baselines by about 10 compared with that from the shortest baselines. With only a few baselines such caution is justified. Obtaining higher resolution effectively means making estimates of the values of the visibility function in areas of the $u-v$ plane beyond $r_{\text {max }}$. With the well-filled $u-v$ plane of QUASAT, an enhancement in resolution of at least $30 \%$ for complex sources can be obtained (A.C.S. Readhead, private communication); a greater enhancement can be expected for strong compact sources.

c) The lower sidelobe level of the synthesized beam in the QUASAT system reduces the effect of residual calibration errors in the data. The major effect of the unknown data errors is to change the size and position of the sidelobe responses in the map away from their expected positions in unpredictable ways; they can thus not be "subtracted out" with full precision. However because of their reduced amplitude compared with the ground-based array the maps will be "de-sensitised" to calibration errors. 
3) Sky coverage - The ground networks planned for operation in Europe, USA and the southern hemisphere by the end of the decade have maximum values of east-west separation of telescopes which are about twice that of the maximum north-south separation. In the equatorial band $|\delta|<30^{\circ}$, the $u-v$ tracks become progressively more linear, so that less of the $u-v$ plane is filled, and in addition the synthesized beam becomes more elongated north-south, reducing the resolution in that direction by up to a factor of two. Since half of the sky is located in this band, this is a substantial problem. The QUASAT VLBI system creates north-south spacings comparable to the east-west whatever the declination of the source, and thus provides excellent $u-v$ coverage over the whole sky.

4) Rapid Mapping - Since a single pass of the QUASAT antenna working with a ground array results in fair $u-v$ coverage a crude map can be constructed from a single pass alone. This property is likely to prove useful for monitoring the structural evolution of flaring systems whose structure can vary significantly in time periods of less than a day (e.g. SS433).

\section{THE QUASAT MISSION}

\section{Summary}

The proposed mission is a free-flying spacecraft carrying a $15 \mathrm{~m}$ radio frequency antenna in an elliptical orbit with perigee of about $4000 \mathrm{~km}$ and apogee of about $15000 \mathrm{~km}$. The space-borne antenna will observe radio sources at frequencies of 22,5 or $1.7 \mathrm{GHz}$ in conjunction with networks of antennas on the ground, and relay the intermediate frequency data via the equivalent of a $40 \mathrm{Mbit} / \mathrm{s}$ (or perhaps a 100 Mbits/sec) link directly to telemetry stations (NASA DSN or ESA networks) on the ground. A clock reference for the antenna in space, stable to 2 or $3 \times 10^{-14}$, will be based on hydrogen maser oscillators on the ground and relayed directly to the satellite from the telemetry stations in turn. All communication with the space VLBI antenna will be through the telemetry station network. The two way link provide rangerate data for orbit determination.

After transmission to the ground, the IF data will be recorded on magnetic tape on the ground in exactly the same way as for the ground based elements of the array. The tapes will then be brought together at the central processing facility (CPF) of the European, US or southern hemisphere VLB array and correlated by local CPF staff. After correlation and calibration, the data will be sent to the appropriate principal investigators. A block diagram of the system is shown in Figure 2.

The mission operational lifetime is expected to be at least two years. 


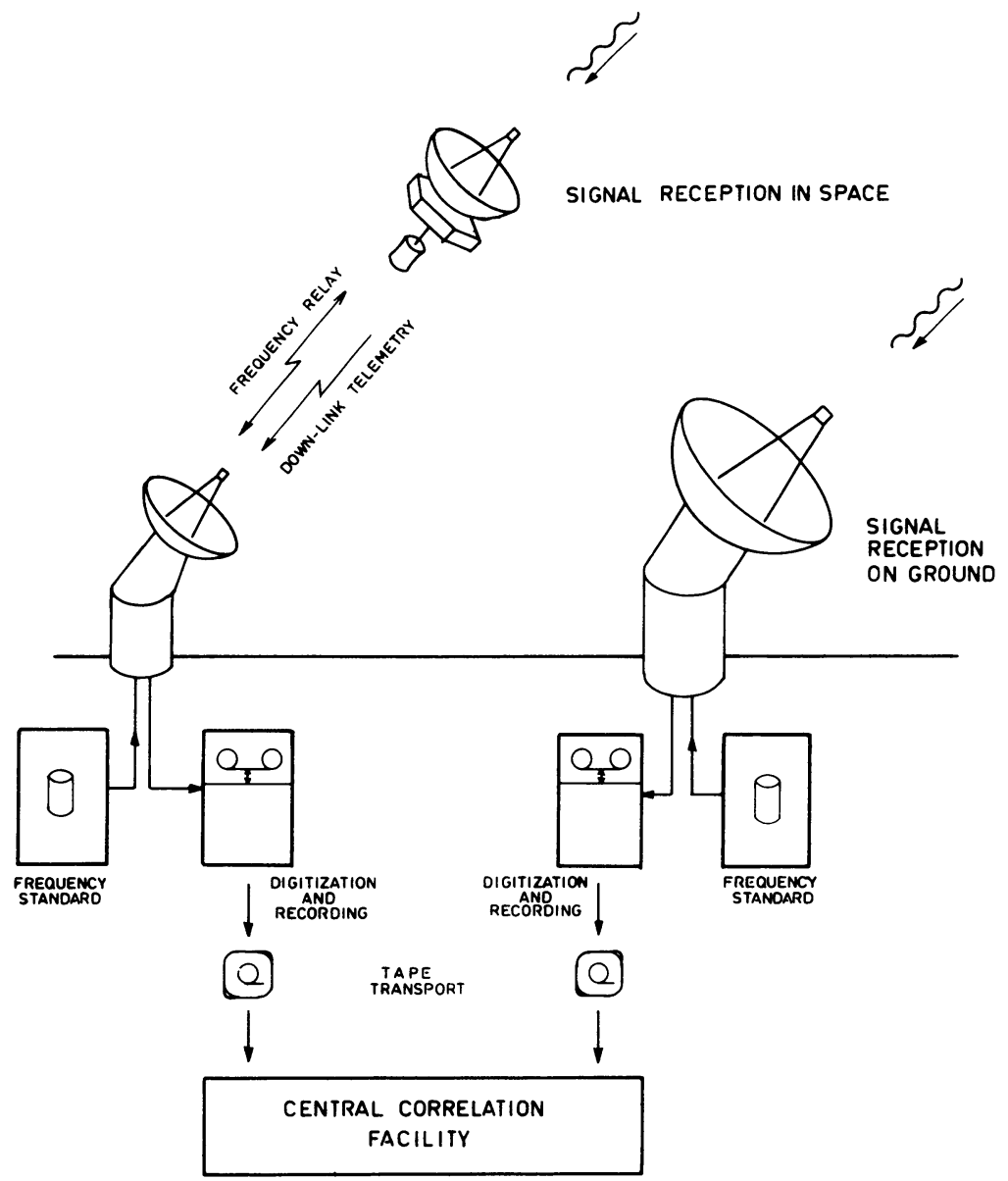

Figure 2 Block diagram of QUASAT VLBI system

Requirements on the Mission

The following are some of the technical requirements on the mission derived from the QUASAT proposal.

Receivers. Observation frequencies are to be $\mathrm{K}$-band $22 \mathrm{GHz}$, C-band $5 \mathrm{GHz}$, and L-band $1.7 \mathrm{GHz}$; simultaneous observation at two frequencies is desired. The receivers should be passively cooled so that the system temperature is $\leqslant 200 \mathrm{~K}$ at $\mathrm{K}$-band, $\leqslant 75 \mathrm{~K}$ at C-band and $\leqslant 40 \mathrm{~K}$ at L-band.

Polarisation. The QUASAT receivers must detect both hands of circular polarisation simultaneously at $1.7,5$ and $22 \mathrm{GHz}$.

I.F. System. The I.F. bandwidth should be at least $20 \mathrm{MHz}$, but the system should be capable of carrying $50 \mathrm{MHz}$ through the I.F. chain for extra sensitivity in the event that the required spectrum space can be arranged. A dual channel IF system is desired to allow for simultaneous 
observation at 2 frequencies or with both hands of circular polarisation.

Desired Orbit Parameters. The orbit semi-major axis must be large enough to provide space-ground baselines long enough to significantly improve on the resolution obtainable from earth, but not so large that substantial holes in the $\mathrm{U}-\mathrm{V}$ plane coverage result. A reasonable goal is to provide baselines of at least $20,000 \mathrm{~km}$. Therefore the height of apoapsis should be at least $15,000 \mathrm{~km}$. The periapsis of the orbit must be low enough to provide intermediate length space-ground baselines down to intercontinental distances. Thus the height of periapsis should be within $5000 \mathrm{~km}$. The inclination of the orbit should be $\sim 45^{\circ}$ so that observations in directions $\pm 45^{\circ}$ from the perpendicular to the orbit plane will cover the bulk of the entire sky as the orbit plane precesses. Preliminary analysis indicates a range of $45^{\circ}$ to $65^{\circ}$ is acceptable for the inclination.

Orbit knowledge. Determination of the orbit should be made with errors not greater than $100 \mathrm{~m}$ in spacecraft position, $10^{-2} \mathrm{~m} / \mathrm{sec}$ in spacecraft velocity and $2 \times 10^{-7} \mathrm{~m} / \mathrm{sec}^{2}$ in spacecraft acceleration so that the residual fringe rates and delays on QUASAT baselines can be brought within standard windows in the central processors. Corrections in real time for the several hundred $\mathrm{kHz}$ fringe rates on QUASAT baselines will then be possible by offsetting an LO synthesizer at the telemetry station.

It is proposed that the even more accurate orbit knowledge required for phase referencing observations (position errors - observing wavelength) be determined, post-hoc, from the radio astronomy data itself.

Mapping time. The typical mapping period will be 24 to 48 hours, but depending on the scientific goal, some observations will last only a few hours.

Observing Direction. Typical observing directions will be approximately perpendicular to the orbit plane.

\section{EXFECTED SCIENTIFIC PERFORMANCE OF QUASAT}

\section{U-V Coverage with the proposed orbit}

Figure 3 shows the $u-v$ coverage resulting from 24 hours of observation with QUASAT in conjunction with (a) the European array plus affiliate stations, and (b) the US dedicated array, for a source at $\delta=$ $60^{\circ}$. The coverage within a radius of $\sim 8000 \mathrm{~km}$ of the $U-V$ plane origin results almost exclusively from the ground array alone while the coverage outside that radius results almost exclusively from baselines to the satellite. 

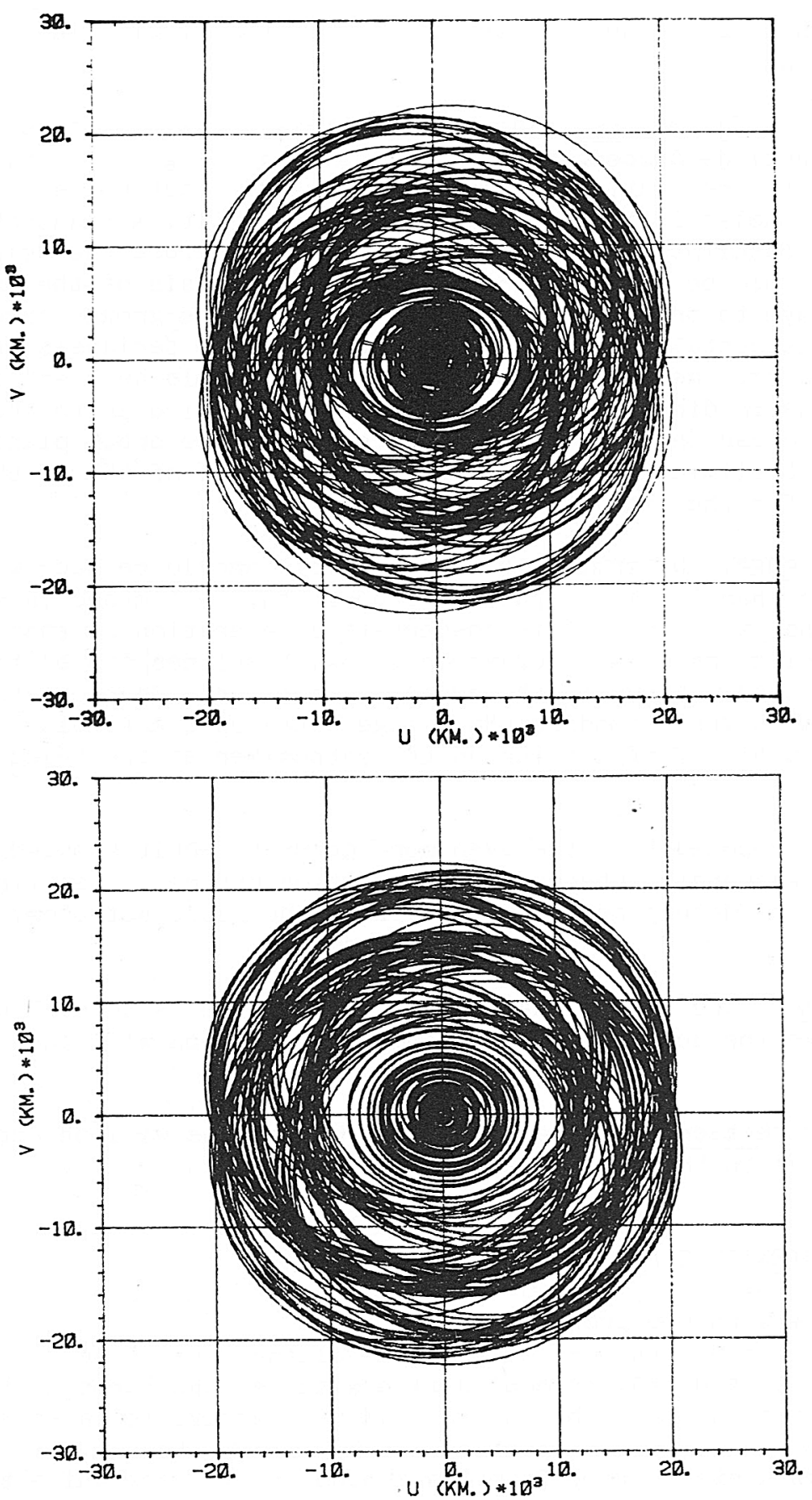

Figure 3 U-V coverage for a source at $\delta=60^{\circ}$ with QUASAT and (a) the US dedicated array and (b) the European VLBI Network and affiliate stations. 
Linear resolution

Observations at $1.35 \mathrm{~cm}$ will be made with a beam of between 60 and $80 \mathrm{microarcsec}$. This corresponds to

1 A.U. at the Galactic Centre

$2 \times 10^{16} \mathrm{~cm}$ (10 light days) at M87

$10^{17} \mathrm{~cm}$ (50 light days) at NGC1275

$1.5 \times 10^{18}(0.5$ parsec $)$ at a redshift of 1 ,

assuming $\mathrm{H}_{\mathrm{O}}=75 \mathrm{~km} \mathrm{~s}^{-1} \mathrm{Mpc}^{-1}$ and $\mathrm{q}_{0}=0.05$

Sensitivity

With the system parameters for QUASAT given in the previous section, and assuming standard parameters for ground radio telescopes, a 100 to 1 dynamic range map should be achieved for $250 \mathrm{mJy}$ sources at $1.35 \mathrm{~cm}$ and $50 \mathrm{mJy}$ sources at 6 and $18 \mathrm{~cm}$ after a 24 hour observation. Source count data indicate that - 1000 compact sources should be mapable at $1.35 \mathrm{~cm}$ and some 10000 at 6 and $18 \mathrm{~cm}$. In a 2 year mission, some 250

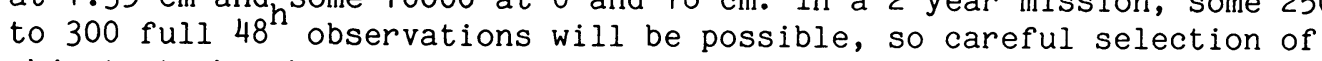
objects to be observed will be necessary.

Field of view

Four factors affect the field of view of the QUASAT system: (a) holes in the $u-v$ plane coverage, (b) integration time per data point in relation to the ( $\leqslant 7 \mathrm{~km} \mathrm{~s}^{-1}$ ) motion of the satellite through the aperture plane, (c) the bandwidth of the individual IF channels, (d), the maximum map size that can be handled by the image processing system. Factors (b) and (c) individually lead to a worst-case field of view of 20 milli-arcsec (with 10\% decorrelation at the field edge) for an integration time of 10 seconds, and a bandwidth of $64 \mathrm{MHz}$ respectively. The latter does not pose a problem, but the former does, since typical integration times to detect fringes on weak sources will be 100 to 300 seconds. However it may be that use of the global fringe fitting algorithm (Schwab and Cotton 1982) for sources which have a compact component in the field of view determined by the 100 to 300 second integration, will allow a wider field of view to be obtained, perhaps as large as that corresponding to integration times of 1 second. This is under study at present. One should also note that a $1024 \times 1024$ map at $1.35 \mathrm{~cm}$ covers a field of view of $20 \mathrm{milli}$-arcsec; however computer limitations to the size of maps are likely to diminish in the next 10 years.

\section{MISSION OPTIONS AND ALTERNATIVES}

Although the general profile of the QUASAT mission is now established there are a number of aspects which will be reviewed for their potential impact on the mission. A partial list of these follow: consequences of a 5 to 10 year mission, $50 \mathrm{MHz}$ link bandwidth, analogue or digital link, cryogenically cooled receivers, on-board stable oscillators, alternative modes for down linking of telemetry (e.g. TDRSS), phase referencing strategies, astrometry and geodesy applications, other receiving frequencies (e.g. 43 and $0.408 \mathrm{GHz}$ ). 
Work has already begun on defining various aspects of the project in more detail. In the USA, A.C.S. Readhead, R.A. Preston and B.F. Burke are leading a group at Caltech and JPL studying the image formation properties of the QUASAT mission, whilst in Sweden R.S. Booth and B.O. Rönnäng (Onsala Space Observatory) are coordinating a preliminary study of the characteristics of the radio astronomy feeds on QUASAT. QUASAT Study Teams have been formed in Europe and the USA which will act as consultants to the ESA and NASA Assessment Studies. A QUASAT Workshop is planned for Summer 1984.

These activities all point to a growing interest being taken in QUASAT in the astronomical community and in the space agencies, an interest that needs to evolve into strong support at national and international level to ensure selection of the mission in three or four years time.

\section{REFERENCES}

Anderson, B. et al (1982) Mission Proposal to the European Space Agency. Burke, B.F. (1982) Astronautics and Aeronautics, October issue. Morgan, S.H. et al (1982) NASA Doc. TM-82491. Preston, R.A. et al (1976) Bull. Amer. Astron. Soc. 8 (No. 4). Preston, R.A. et al (1983) Proc. Conf. on VLBI Techniques, Toulouse p. 417.

Schwab, F.R., and Cotton, W.D. (1983) Astron. J. 88, 688. 